\title{
Análise de Desempenho da Distribuição de Workflows Científicos em Nuvens com Restrições de Confidencialidade*
}

\author{
Rodrigo A. P. Silva ${ }^{1}$, Esther Pacitti ${ }^{2}$, Yuri Frota ${ }^{1}$, Daniel de Oliveira ${ }^{1}$ \\ ${ }^{1}$ Universidade Federal Fluminense (UFF) \\ \{rprado, yuri, danielcmo\}@ic.uff.br
}

${ }^{2}$ INRIA,University of Montpellier, CNRS, LIRMM, França

Esther.Pacitti@inria.fr

\begin{abstract}
Clouds provide an on-demand environment that allows scientists to migrate their local experiments to an elastic environment. Experiments are modeled as scientific workflows, and many of them are computing and data-intensive. The storage of these data is a concern, as confidentiality can be compromised. Malicious users may infer knowledge of the results and structure of workflows. Data dispersion and encryption can be adopted to increase confidentiality, but these mechanisms cannot be adopted uncoupled from workflow scheduling, at the risk of increasing execution time and financial costs. In this paper, we present SaFER (workflow Scheduling with conFidEntity pRoblem), a scheduling approach that considers data confidentiality constraints.
\end{abstract}

Resumo. As nuvens fornecem um ambiente sob demanda que permite que cientistas migrem seus experimentos locais para um ambiente elástico. Os experimentos são modelados como workflows científicos, e muito deles são intensivos em computação e produção de dados. O armazenamento desses dados preocupa, uma vez que a confidencialidade pode ser comprometida. Usuários maliciosos podem inferir conhecimento dos resultados e da estrutura dos workflows. Dispersão dos dados e criptografia podem ser adotados para aumentar a confidencialidade, mas esses mecanismos não pode ser adotados de forma desacoplada ao escalonamento do workflow, sob o risco de aumentar o tempo de execução e despesas. Nesse artigo, apresentamos a SaFER (workflow Scheduling with conFidEntiality pRoblem), uma abordagem de escalonamento que considera restrições de confidencialidade dos dados.

\section{Introdução}

Os workflows científicos (doravante denominados apenas como workflows) são um arcabouço para modelar as etapas de uma simulação científica [de Oliveira et al. 2019]. Essas etapas envolvem a execução de múltiplas aplicações com parâmetros de entrada e de saída. Os workflows são modelados como grafos dirigidos, nos quais os nós representam as atividades (i.e., associadas à um programa, script, etc.) e os arcos representam as dependências de dados entre as atividades [Deelman et al. 2015]. Denominamos ativação a execução de uma atividade que consome um dado específico (de entrada ou produzido pelo próprio workflow). Esses workflows geralmente são modelados e executados usando mecanismos complexos chamados de Sistemas de Gerência de Workflows (SGWfs). Os

\footnotetext{
${ }^{*}$ Os autores agradecem ao CNPq, CAPES a FAPERJ por financiarem parcialmente esse trabalho.
} 
SGWfs apoiam a composição, a execução, o monitoramento e a captura de dados de proveniência [Freire et al. 2008] (histórico de execuções para fins de reprodutibilidade) dos workflows. Em muitos domínios da ciência, como a biologia, a química e a astronomia [de Oliveira et al. 2019] é comum que um mesmo workflow seja executado múltiplas vezes até que o cientista possa confirmar ou refutar uma hipótese. Essas múltiplas execuções, aliadas ao grande volume de dados a ser processado, requerem ambientes de processamento de alto desempenho ou de processamento escalável em uso de dados, como clusters de máquinas commodity e supercomputadores, para que os workflows produzam resultados em tempo hábil [Deelman et al. 2015].

Muitos cientistas migraram seus workflows para nuvens [González et al. 2009], uma vez que as nuvens de computadores oferecem uma ampla variedade de recursos sob demanda, desde máquinas virtuais (VMs), áreas de armazenamento, etc. Além disso, as nuvens oferecem elasticidade, o que pode ser valioso para a execução de workflows em larga escala. Muitos SGWfs permitem a execução de workflows em nuvens como o Pegasus [Deelman et al. 2015] e o SciCumulus [de Oliveira et al. 2010]. Para permitir a execução dos workflows de forma otimizada, cada ativação necessita ser escalonada para uma VM. Logo, o problema de escalonamento se torna decidir em qual VM executar cada uma das ativações do workflow. O escalonamento de ativações de workflows é um conhecido problema NP-Difícil. Os SGWfs já oferecem uma série de algoritmos de escalonamento que exploram características das nuvens. Ao executar workflows na nuvem, os SGWfs existentes comumente se baseiam em áreas de armazenamento compartilhado para gravar os dados produzidos (e.g., chamaremos essas áreas de buckets), e.g., o Pegasus provê mecanismos para armazenar dados no Amazon S3. Da mesma forma, o SciCumulus usa o $s 3 f s 3$ (sistema de arquivos compartilhado sobre o S3) para armazenamento e recuperação de dados. Embora a utilização de serviços de armazenamento na nuvem facilite a gerência dos dados produzidos e consumidos de um workflow, a confidencialidade dos resultados é ainda uma questão aberta [Ennajjar et al. 2017]. Nos últimos anos, muitos cientistas evitaram migrar seus experimentos para a nuvem por questões de confidencialidade. Os arquivos produzidos e consumidos por um workflow podem conter dados confidenciais, e, se os mesmos forem armazenados em conjunto e um usuário malicioso tiver acesso ao bucket, o mesmo pode inferir seus resultados, o que não é desejável.

Para ilustrar esse problema, consideremos um fragmento do workflow Montage [Deelman et al. 2015], apresentado na Figura 1. O Montage cria uma imagem em mosaico a partir de múltiplas imagens astronômicas menores. Apesar do Montage possuir 10 atividades, na Figura 1, apresentamos somente duas (mProjectPP, em azul, e mDiffFit, em amarelo). Cada atividade possuem ativações associadas, cada uma consumindo arquivos de entrada diferentes. Por exemplo, três ativações de $m P r o j e c t P P$ são apresentadas na Figura 1 (ID00000, ID00001 e ID00002), cada uma consumindo arquivos de entrada diferentes (somente region.hdr é consumido pelas três ativações). Os arquivos p2mass-atlas-ID00000s-jID00000_area.fits e p2mass-atlas-ID00000s-jID00000.fits são gerados pela ativação ID00000 (da atividade $m P$ roject $P P$ ), que por sua vez consome os arquivos region.hdr e 2mass-atlas-ID00000s-jID00000.fits. Caso os dois arquivos produzidos sejam armazenados em conjunto com os arquivos de entrada, um usuário malicioso pode inferir que tipo de transformação foi aplicada nos dados. Logo, p2mass-atlas-ID00000sjID00000_area.fits e p2mass-atlas-ID00000s-jID00000.fits não devem ser armazenados em conjunto. Além disso, não devem ser armazenados em conjunto com os arquivos region.hdr e 2mass-atlas-ID00000s-jID00000.fits. Entretanto, nem todos os arquivos possuem 
restrições de armazenamento entre si. Por exemplo, os arquivos p2mass-atlas-ID00000sjID00000_area.fits e p2mass-atlas-ID00000s-jID00000.fits podem ser armazenado em conjunto com o arquivo p2mass-atlas-ID00000s-jID00001_area.fits, pois não foram gerados pela mesma ativação, e nem a partir dos mesmos arquivos de entrada. Essa definição de quais arquivos serão armazenados em conjunto em um bucket se dá o nome de plano de dispersão. De fato, a dispersão é uma das principais técnicas para garantir confidencialidade de dados em nuvens [Branco-Jr. et al. 2016]. As restrições de armazenamento necessárias para gerar um plano de dispersão podem ser representadas como um grafo de conflito (Figura 1(à direita)), onde cada arco ligando dois arquivos representa um conflito (i.e., esses dois arquivos não devem ser armazenados no mesmo bucket).

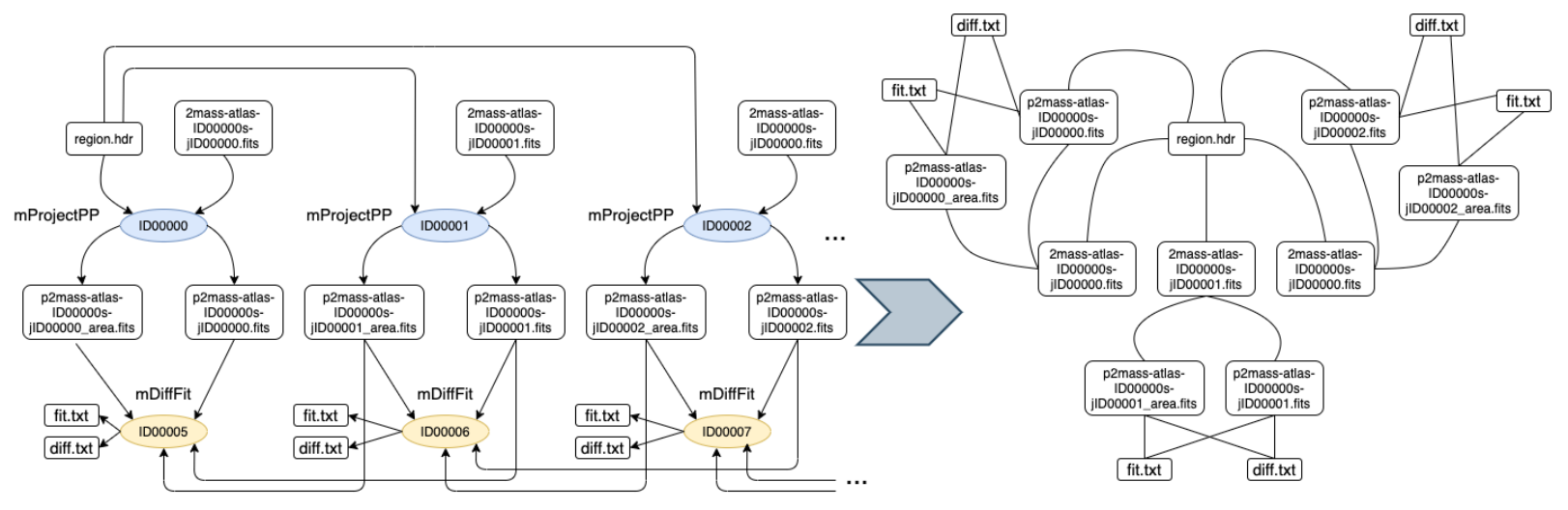

Figura 1. Montage (esquerda) e exemplo de grafo de conflitos do Montage (direita).

Um bom plano de dispersão de dados deve considerar conflitos entre os arquivos e a capacidades/custo de armazenamento nos buckets. Abordagens existentes já tratam do problema de dispersão de dados para garantir a confidencialidade [Guerine et al. 2019]. Porém, tais abordagens dispersam os dados desacoplados do escalonamento das ativações do workflow na nuvem. Essa dispersão sem considerar a VM que a ativação que consumirá os dados irá executar, pode inserir um overhead de transferência desnecessário, e.g., um arquivo pode ser armazenado em um bucket da Amazon S3 em São Paulo e a ativação que o consome executar no Japão. Logo, o plano de dispersão dos dados integrado ao escalonamento das ativações é fundamental. Além disso, [Branco-Jr. et al. 2016] mencionam que a criptografia nas VMs aumenta o nível de segurança nos dados, e devem ser consideradas complementarmente ao plano de dispersão.

Nesse artigo, de forma a garantir a confidencialidade dos dados produzidos e ao mesmo tempo reduzir o tempo total e o custo de execução do workflow, propomos uma abordagem denominada SaFER (workflow Scheduling with conFidEntiality pRoblem) para escalonamento de workflows, que leva em consideração a variedade e a heterogeneidade de VMs na nuvem (por exemplo, diferentes larguras de banda, taxas de transferência e capacidades de processamento), os mecanismos de segurança oferecidos por cada VM (e.g., criptografia) e o grafo de conflito entre os arquivos, todos integrados. Assim, o escalonador do SGWf define a distribuição de ativações nas VMs e o plano de dispersão dos arquivos entre os múltiplos buckets minimizando o tempo total de execução e a chance de um usuário malicioso acessar os dados e inferir o resultado do experimento. As contribuições do presente artigo são: (i) a formulação do problema SaFER como um problema de programação matemática mista, (ii) a definição de um algoritmo de escalonamento de workflows com restrições de confidencialidade, e (iii) uma avaliação experimental da abordagem proposta 
usando benchmarks tradicionais da área de workflows científicos. Esse artigo está organizado em cinco seções além desta introdução. Na Seção 2 apresentamos os trabalhos relacionados. Na Seção 3 é apresentada a formulação matemática, e na Seção 4 a heurística de escalonamento proposta. Na Seção 5, discutimos a avaliação experimental, e na Seção 6 concluímos o presente artigo.

\section{Trabalhos Relacionados}

O escalonamento de ativações de workflows em nuvem é um tópico amplamente discutido na literatura. Existem diversas abordagens de escalonamento que consideram tempo de transferência, custo financeiro, redução de makespan, etc. [Liu et al. 2015]. Entretanto, poucas abordagens focam no escalonamento de workflows considerando questões de confidencialidade e/ou segurança [Sujana et al. 2019, Abazari et al. 2019, Tawfeek and AbdulHamed 2018, Shishido et al. 2018, Guerine et al. 2019]. A abordagem OPTIC [Guerine et al. 2019] propõe uma heurística para dispersão de dados produzidos por workflows em buckets de provedores de nuvem. Apesar de representar um avanço no que tange questões de confidencialidade de dados, a OPTIC não considera o escalonamento do workflow para planejar a dispersão dos dados, i.e., um dado pode ser armazenado em um bucket geograficamente distante da VM em que será processado. Isso pode ser um problema tanto em termos de makespan (já que o tempo de transferência será impactado) quanto em termos financeiros (custos de transferência e pagamento do tempo em que a VM se mantém inativa aguardando a transferência dos dados.

Por outro lado, existem trabalhos que propõem algoritmos de escalonamento de workflows que consideram questões de segurança no processo. [Shishido et al. 2018] propõem uma abordagem de escalonamento de workflows em nuvem que considera restrições de níveis de segurança para as VMs envolvidas na execução, i.e., o algoritmo só executa determinadas ativações em VMs que oferecem os requisitos de segurança necessários. Apesar de ser um avanço [Shishido et al. 2018] não consideram questões de dispersão de dados, i.e., o requisito de segurança é considerado no processamento da ativação, mas não onde os dados são armazenados. De forma similar, [Tawfeek and AbdulHamed 2018] resolve o problema de escalonamento de workflows em nuvem por meio de um algoritmo genético que considera as restrições de segurança, prazo e custo. Assim como o trabalho de [Shishido et al. 2018], as restrições de segurança não consideram questões de armazenamento de dados. [Sujana et al. 2019] e [Abazari et al. 2019] propõem abordagens de escalonamento multi-critério que consideram as demandas de segurança das ativações e as interações na distribuição de ativações seguras na nuvem. De maneira complementar, o i-OBJECT é uma abordagem que visa aumentar o nível de confidencialidade dos dados usando fragmentação, decomposição e dispersão de informações para dividir os dados em partes irreconhecíveis e armazená-los hosts distribuídos na nuvem [Branco-Jr. et al. 2016].

\section{Formulação Matemática}

O problema tratado pela abordagem SaFER pode ser definido como um problema de programação matemática mista como descrito a seguir. Seja $N$ o conjunto de ativações de um workflow a serem executadas. Vamos definir $\bar{M}=M \cup B$ como o conjunto de todos os recursos computacionais envolvidos na execução, onde $M$ é o conjunto de todas as VMs com capacidade de processamento/armazenamento local, e $B$ é o conjunto de buckets que possuem apenas capacidade de armazenamento (i.e., não processam dados). Cada VM 
$j \in M$ possui uma capacidade de armazenamento máxima $\mathrm{cm}_{j}$ e um custo financeiro de $c_{j}^{M}$ (por unidade de tempo). Similarmente, cada bucket $j \in B$ possui uma faixa de intervalos de uso de dados $\left\{0 \ldots\left|L_{j}\right|\right\}$, onde cada intervalo $l \in L_{j}$ possui um tamanho $s m_{j l} \mathrm{e}$ um custo financeiro de uso $c_{j l}^{B}$ ( $L_{0}$ representa que o bucket está vazio). Essa definição de uso de intervalo de dados no bucket segue a organização de provedores como Amazon AWS e Azure. Vamos também definir $D=D_{s} \cup D_{d}$ como o conjunto de todos os dados produzidos/consumidos em uma execução do workflow, onde cada dado $d \in D$ tem tamanho $W(d)$ e pode ser tanto estático $\left(D_{s}\right)$, previamente armazenado no seu recurso computacional de origem $O(d) \in \bar{M}$ (dados de entrada do workflow), ou dinâmico $\left(D_{d}\right)$, i.e., o dado será gerado durante a execução do workflow. Além disso, para cada ativação $i \in N$, definimos um conjunto de dados de entrada $\Delta_{i n}(i) \subseteq D$ necessário para sua execução, e um conjunto de dados de saída $\Delta_{\text {out }}(i) \subseteq D_{d}$ gerados pela execução da ativação. Definimos também os requisitos do usuário $c_{\max }$ como o custo financeiro máximo permitido (i.e., budget)), e $t_{\text {max }}$ como o tempo máximo de processamento do workflow (i.e., deadline), onde $T=\left\{1, \ldots, t_{\max }\right\}$ é definido como o conjunto de períodos de tempo. Denotamos $t_{i j}$ como o tempo de processamento da ativação $i \in N$ executada na VM $j \in M$. Similarmente, denotamos $\vec{t}_{d j p}$ como o tempo gasto pela VM $j \in M$ para ler o dado $d \in D$ armazenado no recurso computacional $p \in \bar{M}$, e $\overleftarrow{t}_{d j p}$ como o tempo gasto pela $\mathrm{VM} j \in M$ para escrever o dado $d \in D_{d}$ no recurso computacional $p \in \bar{M}$.

Em relação aos requisitos de confidencialidade do workflow, definimos o grafo de conflito de dados (nesse artigo usamos arquivos como a unidade mínima, mas caso uma fragmentação dos dados fosse aplicada, cada fragmento seria considerado um vértice do grafo) $G_{c}=\left(D, E_{h} \cup E_{s}\right)$, formado pelo conjunto de vértices (dados) $D$ e o conjunto de arestas $E_{h} \cup E_{s}$. O conjunto $E_{h}$ é definido como o conjunto de pares de dados $\left(d_{1}, d_{2}\right)$ onde $d_{1} \in D$ e $d_{2} \in D$ que não podem ser armazenados no mesmo recurso computacional por razões de confidencialidade. Similarmente, o conjunto de arestas $E_{s}$ é definido como o conjunto de pares de dados $\left(d_{1}, d_{2}\right)$ que se forem armazenados no mesmo recurso computacional, uma penalidade de confidencialidade $p_{d_{1} d_{2}}$ deve ser cobrada. Definimos também $R$ como o conjunto dos requisitos de segurança presentes nas ativações do workflow, onde cada requisito $r \in R$ pode variar de intensidade no intervalo $\left\{0 \ldots l_{\max }^{r}\right\}$. Um requisito de segurança pode ser a ativação $i \in N$ requisitar execuções em VMs que ofereçam alto nível de criptografia (o nivel pode ser definido de acordo com as intensidades dos intervalos, explicadas a seguir). Dessa forma, definimos que cada ativação $i \in N$ possui uma necessidade de $l_{\text {task }}^{r i}$ em relação ao requisito $r \in R$, e que cada VM $j \in M$ pode oferecer $l_{v m}^{r j}$ de segurança em relação a este requisito (onde $0 \leq l_{\text {task }}^{r i}, l_{v m}^{r j} \leq l_{\text {max }}^{r}$ ). Dessa forma, podemos definir o grau de exposição máxima de confidencialidade de um escalonamento como $s_{\max }=\sum_{r \in R}|N| \cdot l_{\max }^{r}+\sum_{\left(d_{1}, d_{2}\right) \in E_{s}} p_{d_{1} d_{2}}$. A Tabela 3 descreve o conjunto de variáveis binárias e contínuas do modelo descrito a seguir:

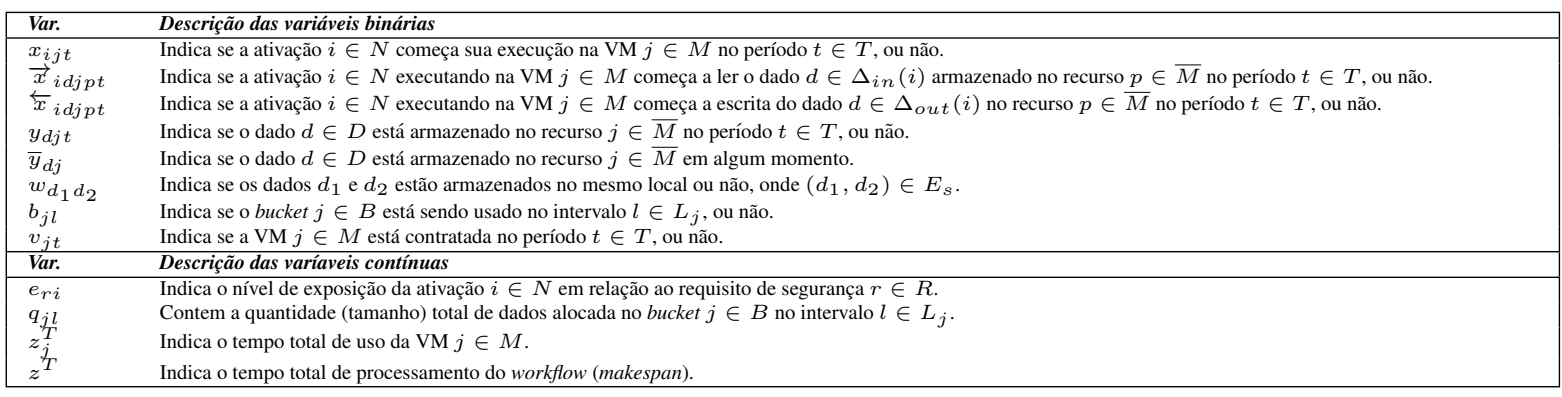


A função objetivo procura minimizar 3 fatores: o makespan (1), o custo financeiro (2) e o nível de exposição de confidencialidade (3), onde os pesos $\alpha_{t}, \alpha_{b}$ e $\alpha_{s}$ definem a relevância de cada um destes 3 objetivos, respectivamente. Além disso, o valor de cada um dos objetivos é normalizado por seus valores máximos definidos por $t_{\max }, c_{\max }$ e $s_{\max }$ respectivamente.

$$
\begin{aligned}
\min & \alpha_{t} \cdot\left(\frac{z^{T}}{t_{\max }}\right)+ \\
& \alpha_{b} \cdot\left(\sum_{j \in M} \frac{c_{j}^{M} z_{j}^{T}}{c_{\max }}+\sum_{j \in B} \sum_{l \in L_{j}} \frac{c_{j l}^{B} q_{j l}}{c_{\max }}\right) \\
& \alpha_{s} \cdot\left(\sum_{r \in R} \sum_{i \in N} \frac{e_{\text {ri }}}{s_{\max }}+\sum_{\left(d_{1}, d_{2}\right) \in E_{s}} \frac{p_{d_{1} d_{2} w_{d_{1} d_{2}}}}{s_{\max }}\right)
\end{aligned}
$$

As Restrições (4) garantem que cada ativação será executada, enquanto que as Restrições (5) e (6) obrigam que cada operação de leitura e escrita deve ser realizada.

$$
\begin{aligned}
& \sum_{j \in M} \sum_{t \in T} x_{i j t}=1, \\
& \sum_{\substack{j \in M \\
p \in \bar{M}}} \sum_{t \in T} \vec{x}_{i d j p t}=1, \\
& \sum_{\substack{j \in M \\
p \in \bar{M}}} \sum_{t \in T} \overleftarrow{x}_{i d j p t}=1,
\end{aligned}
$$

As Restrições (7) garantem que cada dado $d \in \Delta_{\text {out }}(i)$ só pode ser escrito, se a ativação $i$ foi executada anteriormente. Além disso, as Restrições (8) obrigam que cada dado $d$ não possa ser escrito antes da execução da ativação $i$ (responsável por sua escrita).

$$
\begin{aligned}
& \overleftarrow{x}_{i d j p t} \leq \sum_{q=1}^{t-t_{i j}} x_{i j q} \\
& \overleftarrow{x}_{i d j p t}=0
\end{aligned}
$$$$
\forall i \in N, \forall d \in \Delta_{\text {out }}(i), \forall j \in M, \forall p \in \bar{M}, \forall t=\left(t_{i j}+1\right) \cdots T_{M} \text { (7) }
$$$$
\forall i \in N, \forall d \in \Delta_{\text {out }}(i), \forall j \in M, \forall p \in \bar{M}, 1 \leq t \leq t_{i j}(8)
$$

As Restrições (9) estabelecem que uma ativação só pode ser executada se todas suas leituras de dados de entrada foram anteriormente concluídas.

$x_{i j t} \leq \sum_{p \in \bar{M}} \sum_{q=1}^{t-\vec{t}_{d j p}} \vec{x}_{i d j p q}$,

$\forall i \in N, \forall d \in \Delta_{i n}(i), \forall j \in M, \forall t \in T, \operatorname{such}\left(t-\vec{t}_{d j p}\right) \geq 1$ (9)

As Restrições (10) garantem que apenas uma ação ativa (execução, leitura ou escrita) possa ser realizada em cada período de tempo em cada VM. Por outro lado, as Inequações (11) definem que ações passivas (um dado ser lido ou ser escrito) podem ser realizadas em paralelo. Note que se uma ação (ativa ou passiva) é realizada por uma VM $j$ no período $t$, a variável de contratação $v_{j t}$ terá valor 1 , indicando que a máquina está contratada.

$$
\begin{aligned}
& \sum_{i \in N} \sum_{q=\max }^{t}\left(1, t-t_{i j}+1\right) \\
& \sum_{i \in N} \sum_{d \in \Delta_{i n}(i)} \sum_{p \in \bar{M}}+\sum_{i \in N=\max } \sum_{\left(1, t-\vec{t}_{d j p}+1\right)} \sum_{d \in \Delta_{\text {out }}(i)} \sum_{p \in \bar{M}} \vec{x}_{r=\max } \sum_{\left(1, t-\overleftarrow{t}_{d j p}+1\right)}^{t} \overleftarrow{x}_{i d j p r}+ \\
& \sum_{i \in N} \sum_{d \in \Delta_{\text {out }}(i)} \sum_{p \in M} \sum_{r=\max } \sum_{\left(1, t-\overleftarrow{t}_{d p j}+1\right)}^{t} \overleftarrow{x}_{i d p j r}+ \\
& \sum_{i \in N} \sum_{d \in \Delta_{i n}(i)} \sum_{p \in M} \sum_{r=\max }^{t} \vec{x}_{\left(1, t-\vec{t}_{d p j}+1\right)} \vec{x}_{i d p j r} \leq|M| \cdot v_{j t},
\end{aligned}
$$


As Restrições (12) estabelecem que não existem dados dinâmicos gerados no início da execução, enquanto que as Restrições (13) e (14) estabelecem que os dados estáticos estão armazenados em seus respectivos recursos origem.

$y_{d j 1}=0$,

$y_{d j 1}=0$,

$\forall d \in D_{d}, \forall j \in \bar{M}(12)$

$y_{d j t}=1$,

$\forall d \in D_{s} \mid j \in(\bar{M} \backslash O(d))(13)$

$\forall d \in D_{s} \mid j \in O(d), \forall t \in T$ (14)

As Restrições (15) e (16) conectam as variáveis de armazenamento y com as variáveis de escrita $\overleftarrow{x}$ e as de leitura $\vec{x}$, garantindo uma leitura e escrita consistente com os dados armazenados tanto em VMs quanto em buckets $(\bar{M})$.

$$
\begin{aligned}
& y_{d p(t+1)}=y_{d p t}+\sum_{j \in M} \overleftarrow{x}_{i d j p\left(t-\overleftarrow{t}_{d j p}+1\right)} \\
& \sum_{j \in M} \vec{x}_{i d j p t} \leq y_{d p t}
\end{aligned}
$$

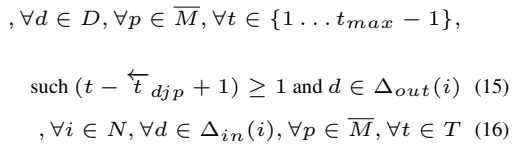

A capacidade de armazenamento das máquinas é limitado pelas Restrições (17). As Restrições (18) conectam a última operação de escrita em cada máquina com a variável do tempo total de execução do workflow $z^{T}$. Além disso, as Restrições (19) relacionam as variáveis de contratação $v_{j t}$ e $z_{j}^{T}$ com o objetivo de estabelecer o custo financeiro de cada VM $j$, enquanto que as Restrições (20) asseguram que os custos financeiros não ultrapassem o orçamento máximo disponível.

$$
\begin{aligned}
& \sum_{d \in D} y_{d j t} W(d) \leq c m_{j} \\
& \overleftarrow{x}_{i d j p t} \cdot\left(t+\overleftarrow{t}_{d j p}\right) \leq z^{T} \\
& v_{j t} \cdot t \leq z_{j}^{T}, \\
& \sum_{j \in M} c_{j}^{M} z_{j}^{T}+\sum_{j \in B} \sum_{l \in L_{j}} c_{j l}^{B} q_{j l} \leq c_{\max }
\end{aligned}
$$$$
, \forall j \in \bar{M}, \forall t \in T(17)
$$$$
, \forall i \in N, \forall d \in \Delta_{\text {out }}(i), \forall j \in M, \forall p \in \bar{M}, \forall t \in T \text { (18) }
$$$$
\forall j \in M, \forall t \in T(19)
$$

As seguintes restrições operacionais (21) precisam ser satisfeitas: uma ativação $i$ só pode iniciar sua execução se todos seus arquivos de entrada $d \in \Delta_{i n}(i)$ já estiverem disponíveis para leitura.

$\sum_{p \in \bar{M}} \vec{x}_{i d j p t} \cdot\left|\Delta_{i n}(i)\right| \leq \sum_{g \in \Delta_{i n}(i)} \sum_{p \in \bar{M}} y_{g p t}$

$, \forall i \in N, \forall d \in \Delta_{i n}(i), \forall j \in M, \forall t \in T$ (21)

As Restrições (22) conectam as variáveis de armazenamento (temporais) y com as variáveis de armazenamento (atemporais) $\bar{y}$. Restrições (23) asseguram que dados conflitantes não sejam armazenados no mesmo recurso) (arestas fortes). De forma similar, as Inequações (24) garantem que se dois dados conflitantes são armazenados no mesmo recurso, a variável de penalização $w$ terá valor 1 (arestas fracas). As Restrições (25) medem o nível de exposição (risco) de se executar uma ativação em relação aos requisitos de segurança.

$$
\begin{aligned}
& y_{d j t} \leq \bar{y}_{d j} \\
& \bar{y}_{d_{1} j}+\bar{y}_{d_{2} j} \leq 1 \\
& \bar{y}_{d_{1 j} j}+\bar{y}_{d_{2} j} \leq 1+w_{d_{1} d_{2}} \\
& l_{\text {task }}^{r i}-\sum_{j \in M} \sum_{t \in T} l_{v m}^{r j} x_{i j t} \leq e_{r i}
\end{aligned}
$$

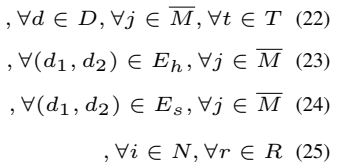


As Equações (26) definem o tamanho total dos dados armazenados em cada bucket. As Restrições (27) estabelecem que apenas um intervalo de armazenamento $l \in L_{j}$ é usado em cada bucket $j \in B$, enquanto as Restrições (28) limitam a quantidade de dados armazenados em cada intervalo no bucket. Note que as Restrições (27) e (29) estabelecem em qual intervalo de uso de dados $\left(b_{j l}\right)$ o bucket se encontra, de acordo com a quantidade de dados total $\left(q_{j l}\right)$ armazenada nele. Por fim, as Restrições (30) impõe que se um intervalo $l \in L_{j} \backslash\{0\}$ do bucket $j \in B$ está em uso, então deve existir pelo menos um dado armazenado no bucket $j$.

$$
\begin{aligned}
& \sum_{l \in L_{j}} q_{j l}=\sum_{d \in D} \bar{y}_{d j} W(d) \\
& \sum_{l \in L_{j}} b_{j l}=1 \\
& \sum_{d \in D} \bar{y}_{d j} W(d) \leq \sum_{l \in L_{j}} s m_{j l} b_{j l} \\
& s m_{j(l-1)} \cdot b_{j l} \leq q_{j l} \leq s m_{j l} \cdot b_{j l} \\
& b_{j l} \leq \sum_{d \in D} \bar{y}_{d j}
\end{aligned}
$$

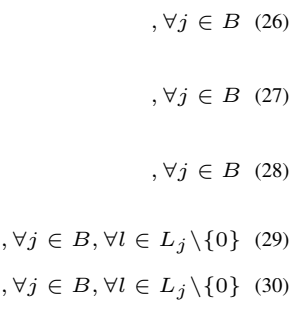

\section{Abordagem Heurística para Escalonamento de Workflows com Restrições de Confidencialidade}

O problema modelado na Seção 3 pode ser resolvido por métodos exatos para instâncias pequenas (10 ativações ou menos). Porém, são impraticáveis para instâncias do mundo real pois consomem muitos recursos computacionais elevando demasiadamente o tempo de execução. As Metaheurísticas são métodos que orquestram uma interação entre procedimentos de melhorias locais e estratégias de nível superior para criar um processo capaz de escapar dos ótimos locais efetuando buscas no espaço de solução [Gendreau et al. 2010], apresentando-se como uma boa alternativa aos métodos exatos. Logo, propomos uma heurística construtiva gulosa e estocástica como parte da abordagem SaFER, denominada SaFER-hcg, para resolver o escalonamento de ativações com restrição de confidencialidade. Nosso objetivo é encontrar boas soluções para instâncias de grande porte.

No Algoritmo 1, construtivo, o laço mais externo (linha 2) itera até que todas as ativações sejam executadas em alguma VM. Nesse laço, é criada uma Lista de Candidatos (LC) combinando cada ativação ainda não escalonada $i \in \bar{N}$ com todas as VMs disponíveis $j \in M$ (linhas 4 a 9). Porém, nem todas as ativações estão prontas para serem adicionadas à LC porque dependem se as dependências de dados foram satisfeitas, i.e., se seus arquivos de entrada já estão disponíveis (linha 6). Para calcularmos o "custo" de cada candidato, o inserimos na solução atual $S$ e calculamos o valor da função objetivo $(F O)$ descrita pelas Equações (1-3). Dentro do cálculo da função objetivo, definimos também os recursos computacionais $W \subseteq \bar{M}$ que irão armazenar os dados de saída da ativação $i$ da seguinte forma: para cada dado $d \in \Delta_{\text {out }}(i)$ selecionamos aleatoriamente $\beta$ recursos computacionais dentre o conjunto $\bar{M}$ e escolhemos o de menor acréscimo ao valor da $F O$ que não irá gerar inviabilidade em relação às restrições de capacidade e confidencialidade para realizar a gravação do dado. Em seguida, ordenamos a LC em função do valor do custo $(F O)$ de cada solução (linha 9) e criamos uma Lista Restrita de Candidatos (LRC) contendo os melhores candidatos usando o parâmetro $\alpha_{L R C}$ (linha 10). Posteriormente, um candidato é selecionado aleatoriamente de LRC e adicionado ao escalonamento atual $S$ (linhas 11 e 12). O procedimento é repetido até que todas as ativações do workflow sejam escalonadas. Dadas as características aleatórias do algoritmo, o procedimento construtivo pode gerar diferentes soluções para um mesmo dado de entrada, i.e., para uma mesma instância do workflow. 
Em nossos experimentos, executamos o algoritmo da SaFER-hcg 100 vezes armazenando, sempre, o melhor resultado encontrado.

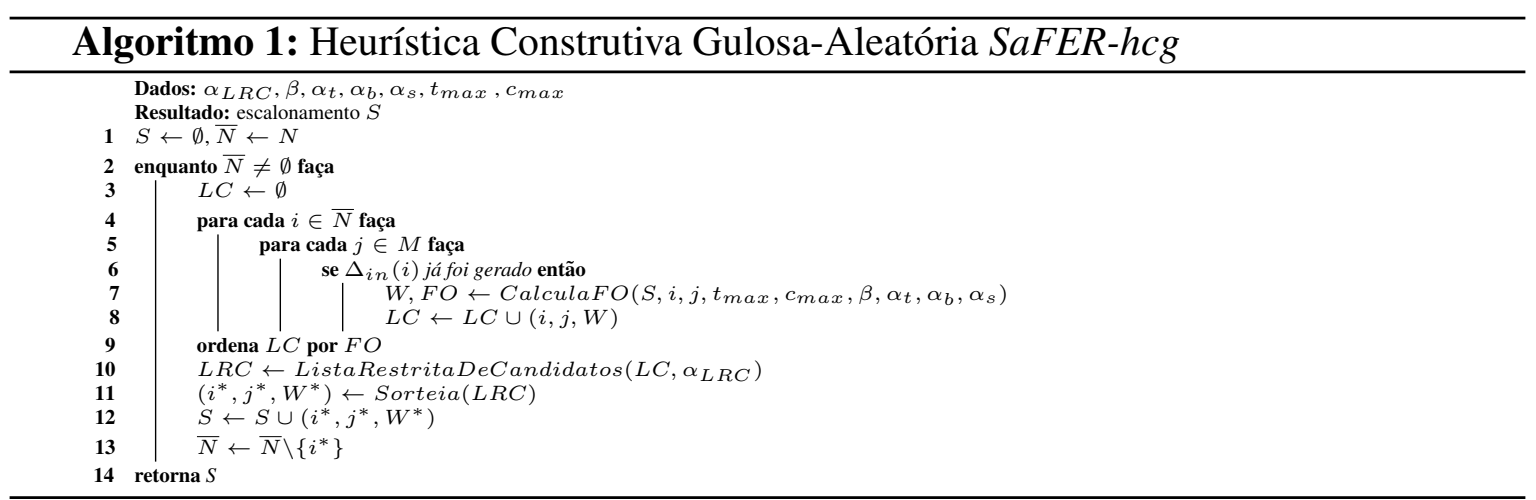

Tabela 1. Detalhes das instâncias utilizadas nos testes experimentais

\begin{tabular}{lrrrr}
\hline Instância & \#Ativações & \#Arquivos & $\mathbf{t}_{\max }$ & $\mathbf{c}_{\max }$ \\
\hline Sintética_5_A & 2 & 3 & 32 & 120 \\
Sintética_5_B & 2 & 3 & 32 & 120 \\
Sintética_5_C & 1 & 4 & 40 & 80 \\
Sintética_10_A & 4 & 6 & 50 & 168 \\
Sintética_10_B & 3 & 7 & 36 & 198 \\
Sintética_10_C & 4 & 6 & 60 & 440 \\
Sintética_15_A & 5 & 10 & 40 & 154 \\
Sintética_15_B & 5 & 10 & 30 & 54 \\
Sintética_15_C & 5 & 10 & 40 & 136 \\
CyberShake_30 & 30 & 49 & 400 & 200 \\
CyberShake_50 & 50 & 79 & 500 & 250 \\
CyberShake_100 & 100 & 154 & 600 & 300
\end{tabular}

\begin{tabular}{lrrrr} 
Instância & \#Ativações & \#Arquivos & $\mathbf{t}_{\max }$ & \multicolumn{1}{c}{$\mathbf{c}_{\max }$} \\
\hline Epigenomics_24 & 24 & 38 & 5.000 & 1.500 \\
Epigenomics_46 & 46 & 71 & 10.000 & 3.000 \\
Epigenomics_100 & 100 & 152 & 50.000 & 30.000 \\
Inspiral_30 & 30 & 47 & 1.500 & 1.000 \\
Inspiral_50 & 50 & 77 & 2.000 & 1.500 \\
Inspiral_100 & 100 & 151 & 3.000 & 2.000 \\
Montage_25 & 25 & 54 & 250 & 125 \\
Montage_50 & 50 & 107 & 500 & 250 \\
Montage_100 & 100 & 215 & 1.000 & 500 \\
Sipht_30 & 29 & 963 & 3.500 & 2.000 \\
Sipht_60 & 58 & 1.100 & 4.000 & 2.500 \\
Sipht_100 & 97 & 1.223 & 6.000 & 3.500 \\
\hline
\end{tabular}

\section{Avaliação Experimental}

Nesta seção, comparamos a solução heurística SaFER-hcg (Seção 4) com a solução exata fornecida pelo modelo matemático (i.e., baseline). Para compararmos as soluções, foram criadas 9 instâncias sintéticas de execuções de workflows, pequenas o suficiente para que o método exato encontre alguma solução. Além disso, executamos a solução heurística SaFER-hcg para instâncias reais de workflows com características distintas e inviáveis para execução com o método exato. De forma a garantir a reprodutibilidade da abordagem proposta, o código-fonte e as configurações de ambiente e dados de entrada se encontram disponibilizados em https://github.com/UFFeScience/Wf-Security. Os métodos heurísticos e exatos foram implementados com GCC 4.8.5 e IBM ILOG CPLEX 12.8; executados em um computador com processador Intel@i5-7300U $2.60 \mathrm{GHz}$, com 16GB de RAM e SO Linux Fedora 31. Além disso, cada teste foi repetido 10 vezes (com sementes aleatórias diferentes) armazenando-se a médias dos resultados. O valor dos parâmetros de entrada $\alpha_{L R C}=0.5$ e $\beta=4$ foram definidos de forma empírica e as ponderações da função objetivo $\alpha_{t}, \alpha_{b}, \alpha_{s}$ foram definidas como $0,3,0,3$ e 0,4 , respectivamente, baseados na experiência em trabalhos anteriores do grupo [Teylo et al. 2017].

Considerando o recursos computacionais necessários para a viabilidade da solução, utilizamos seguintes parâmetros para execução dos workflows: 4 VMs com diferentes capacidade de armazenamento (80, 120, 160 e $200 \mathrm{GBs);} \mathrm{slowdown} \mathrm{(quanto} \mathrm{menor,} \mathrm{maior} \mathrm{o} \mathrm{po-}$ der de processamento) (1,53, 0,77, 0,38 e 0,19); velocidade do enlace (4, 9, 10 e $10 \mathrm{Mbps})$; e o custo, por minuto, de processamento em dólares (US\$ 0,02, US\$ 0,09, US\$ 0,16 e US\$ 
0,33). Em se tratando da quantidade de buckets, definimos 2 para as instâncias sintéticas e variados números para as demais instâncias (valores apresentados a seguir). Quanto a capacidade de armazenamento, velocidade do enlace e preço cobrado por GBs de dados armazenados de cada bucket, definimos como 50 TB, 25 Mbps e US\$ 0,023, respectivamente. Para as instâncias públicas de workflows tradicionais (i.e., benchmarks) foram utilizadas as seguintes: (i) Montage, que cria uma imagem em mosaico a partir de fotos astronômicas menores, (ii) Cybershake, usado para identificar terremotos na Califórnia, (iii) Inspiral, usado para identificar ondas gravitacionais, (iv) Epigenomics, para sequenciamento genômico e (v) Sipht, que busca por RNA não transcrito no NCBI. Foram determinadas as seguintes quantidades de buckets para as instâncias CyberShake_30 (o número no nome da instância define a quantidade de ativações a serem escalonadas), CyberShake_50, CyberShake_100, Epigenomics_24, Epigenomics_46, Epigenomics_100, Inspiral_30, Inspiral_50, Inspiral_100, Montage_25, Montage_50, Montage_100, Sipht_30, Sipht_50, Sipht_100: 2, 8, 19, 6, 6, 44, $3,8,9,6,11,28,10,10$ e 16 , respectivamente. A Tabela 1 apresenta os valores $t_{\max }, c_{\max }$, a quantidade de ativações e a quantidade de arquivos (tanto os de entrada quanto os gerados durante a execução do workflow) escolhidos para cada instância utilizada nos experimentos.

Criamos um script para ler os arquivos que contém as instâncias dos workflows (em formato XML) e atribuir restrições (arestas fortes e fracas) entre os arquivos automaticamente, (i.e., o grafo de conflitos). Para o presente experimento, utilizamos a seguinte heurística para criação do grafo de conflitos: (i) arquivos de entrada de uma ativação são penalizados (com um valor padrão de 1) se armazenados junto dos arquivos de saída desta mesma ativação (arestas fracas), (ii) arquivos gerados por ativações em um mesmo nível do workflow (i.e., gerados por ativações "irmãs") não podem ser armazenados juntos (arestas fortes). No caso do Montage, esta última restrição evita tanto que arquivos cabeçalho e corpo sejam armazenados juntos, e que arquivos que componham partes diferentes da solução também sejam armazenados juntos (e.g., dados gerados por diferentes ativações que formam um mosaico). Quanto aos requisitos definidos na Seção 3, utilizamos valores que permitissem que todas as ativações pudessem ser executadas em qualquer VM, pois focamos nas restrições de confidencialidade, uma vez que, requisitos como autenticação ou criptografia são funcionalidades já estudadas em trabalhos anteriores (vide Seção 2).

A Tabela 2 apresenta os resultados das execuções das heurística SaFER-hcg e a solução exata. As colunas Instância, F. O., Makespan, Custo, Confid., Tempo representam, respectivamente, as instâncias de workflows, função objetivo, tempo de execução, custo e risco de exposição, das execuções da heurística SaFER-hcg e do método exato. Para uma melhor interpretação, a coluna Confid. foi normalizada pelo fator $S_{\max }$. Os resultados indicam que o modelo matemático foi capaz de retornar soluções ótimas para todas as instâncias sintéticas, com exceção das instâncias Sintética_15_B e Sintética_15_C que interromperam sua execução após uma hora (tempo limite configurado para a execução do CPLEX). Por outro lado, a SaFER-hcg conseguiu, para as mesmas instâncias, produzir boas soluções com diferença de, em média, $14 \%$ em relação a solução encontrada pelo método exato, mas não conseguiu encontrar a solução ideal para 6 das instâncias sintéticas. Uma explicação para a diferença entre o método exato e a SaFER-hcg é que o método exato permite que as leituras de todas as ativações iniciais ocorram antes de qualquer execução, enquanto que a heurística SaFER-hcg agrega leitura, execução e escrita atomicamente; isso faz com que o recurso que contém os arquivos de entrada tenha que ser alocado por mais tempo. É interessante notar que os tempos de execução para a SaFER-hcg são inferiores aos executados pelo CPLEX (em torno de um centésimo de segundo). 
Considerando que quanto menor os valores de Confid. menor o risco de exposição de dados confidenciais, os valores baixos demonstram a eficiência da abordagem proposta. Por último, somente a heurística $S a F E R$ - hcg conseguiu encontrar soluções para as instâncias maiores em tempo hábil, comprovando, assim, sua importância.

Tabela 2. Avaliação Experimental - SaFER-hcg versus Solução Exata

\begin{tabular}{|c|c|c|c|c|c|c|c|c|c|c|}
\hline \multirow[b]{2}{*}{ Instância } & \multicolumn{5}{|c|}{ Heurística (SaFER-hcg) } & \multicolumn{4}{|c|}{ Solução Exata } & \multirow[b]{2}{*}{ Tempo (s) } \\
\hline & F. O. & Makespan (s) & Custo (US\$) & Confid. & Tempo (s) & F. O. & Makespan (s) & Custo (US\$) & Confid. & \\
\hline Sintética_5_A & 0,25 & 24,00 & 8,32 & 0,00 & 0,01 & 0,25 & 24,00 & 8,32 & 0,00 & 0,16 \\
\hline Sintética_5_B & 0,28 & 27,00 & 9,35 & 0,00 & 0,01 & 0,28 & 27,00 & 9,21 & 0,00 & 1,29 \\
\hline Sintética_10_A & 0,33 & 48,00 & 22,42 & 0,00 & 0,01 & 0,24 & 35,00 & 16,81 & 0,00 & $1.039,48$ \\
\hline Sintética_10_B & 0,28 & 31,00 & 14,24 & 0,00 & 0,01 & 0,26 & 29,00 & 11,68 & 0,00 & 60,68 \\
\hline Sintética_10_C & 0,31 & 58,00 & 24,78 & 0,00 & 0,01 & 0,28 & 53,00 & 22,97 & 0,00 & 18,44 \\
\hline Sintética_15_C & 0,34 & 36,80 & 17,81 & 0,05 & 0,01 & 0,29 & 29,00 & 16,63 & 0,09 & $3.604,57^{*}$ \\
\hline CyberShake_30 & 0,27 & 158,30 & 93,02 & 0,02 & 0,19 & & & & & \\
\hline CyberShake_50 & 0,39 & 294,20 & 172,95 & 0,01 & 0,78 & & & & & \\
\hline CyberShake_100 & 0,46 & 420,40 & 249,78 & 0,01 & 7,15 & & & & & \\
\hline Epigenomics_24 & 0,42 & $2.698,50$ & $1.204,41$ & 0,04 & 0,12 & & & & & \\
\hline Epigenomics_46 & 0,43 & $4.928,40$ & $2.773,21$ & 0,02 & 0,40 & & & & & \\
\hline Inspiral_30 & 0,33 & 130,30 & 68,70 & 0,02 & 0,15 & & & & & \\
\hline Inspiral_50 & 0,35 & 266,60 & 155,43 & 0,01 & 1,59 & & & & & \\
\hline Inspiral_100 & 0,37 & 561,90 & 329,79 & 0,00 & 19,13 & & & & & \\
\hline Sipht_30 & 0,49 & $2.869,70$ & $1.440,52$ & 0,06 & 3,26 & & & & & \\
\hline Sipht_60 & 0,55 & $3.664,10$ & $2.105,33$ & 0,06 & 17,16 & & & & & \\
\hline Sipht_100 & 0,46 & $4.622,00$ & $2.571,43$ & 0,03 & 52,63 & & & & & \\
\hline
\end{tabular}

* A execução foi interrompida após uma hora de processamento.

\section{Conclusões e Trabalhos Futuros}

Diversos experimentos são modelados como workflows. Esses workflows são, geralmente, compostos por programas caixa preta com dependência de dados entre eles. Muitos dados são produzidos durante a execução de workflows de larga-escala. Quando essa execução é feita na nuvem, comumente os SGWfs existentes armazenam os dados produzidos em um storage compartilhado (i.e., bucket). Ao fazer isso, a confidencialidade do experimento pode ser comprometida. Caso um usuário malicioso venha a acessar esses arquivos, existe o risco do mesmo inferir os resultados e/ou a estrutura do experimento. Esse risco deve ser evitado, ou, pelo menos, minimizado. Nesse artigo, formalizamos o problema de escalonamento de ativações de workflows com restrição de confidencialidade, modelando como um problema de otimização combinatória para preservar a confidencialidade dos dados produzidos e armazenados na nuvem. Introduzimos uma formulação matemática e um procedimento heurístico chamado SaFER-hcg para resolver o problema de otimização. A abordagem proposta utiliza um grafo de conflitos para integrar o escalonamento de ativações com o plano de dispersão dos dados determinando as áreas de armazenamento para os arquivos produzidos durante a execução, respeitando os limites de capacidade. A abordagem proposta foi avaliada com uma série de instâncias de workflows sintéticos e reais, e se mostrou promissora no que tange a garantia da confidencialidade ao mesmo tempo em que foi capaz de minimizar o makespan. Como trabalho futuro, pretendemos implementar uma metaheurística (e.g., GRASP) a fim de melhorar a qualidade da solução heurística proposta, além de implementar o SaFER-hcg no SGWf SciCumulus. 


\section{Referências}

Abazari, F., Analoui, M., Takabi, H., e Fu, S. (2019). Mows: multi-objective workflow scheduling in cloud computing based on heuristic algorithm. Simulation Modelling Practice and Theory, 93:119-132.

Branco-Jr., E. C., Monteiro, J. M., Reis, R., e Machado, J. C. (2016). A new mechanism to preserving data confidentiality in cloud database scenarios. In ICEIS, volume 291, pages 261-283. Springer.

de Oliveira, D., Liu, J., e Pacitti, E. (2019). Data-Intensive Workflow Management: For Clouds and Data-Intensive and Scalable Computing Environments. Synthesis Lectures on Data Management. Morgan \& Claypool Publishers.

de Oliveira, D., Ogasawara, E. S., Baião, F. A., e Mattoso, M. (2010). Scicumulus: A lightweight cloud middleware to explore many task computing paradigm in scientific workflows. In IEEE CLOUD 2010, Miami, pages 378-385.

Deelman, E., Vahi, K., Juve, G., Rynge, M., Callaghan, S., Maechling, P., Mayani, R., Chen, W., da Silva, R. F., Livny, M., e Wenger, R. K. (2015). Pegasus, a workflow management system for science automation. FGCS, 46:17-35.

Ennajjar, I., Tabii, Y., e Benkaddour, A. (2017). Securing data in cloud computing by classification. BDCA'17, New York, NY, USA. ACM.

Freire, J., Koop, D., Santos, E., e Silva, C. T. (2008). Provenance for Computational Tasks: A Survey. Computing in Science \& Engineering, pages 20-30.

Gendreau, M., Potvin, J.-Y., et al. (2010). Handbook of metaheuristics, volume 2. Springer.

González, L. M. V., Rodero-Merino, L., Caceres, J., e Lindner, M. A. (2009). A break in the clouds: towards a cloud definition. Comp. Comm. Rev., 39(1):50-55.

Guerine, M., Stockinger, M. B., Rosseti, I., Simonetti, L. G., Ocaña, K. A., Plastino, A., e de Oliveira, D. (2019). A provenance-based heuristic for preserving results confidentiality in cloud-based scientific workflows. FGCS, 97:697- 713.

Liu, J., Pacitti, E., Valduriez, P., e Mattoso, M. (2015). A survey of data-intensive scientific workflow management. J. Grid Comput., 13(4):457-493.

Shishido, H. Y., Estrella, J. C., e Toledo, C. F. M. (2018). Multi-objective optimization for workflow scheduling under task selection policies in clouds. In $C E C$, pages 1-8. IEEE.

Sujana, J. A. J., Revathi, T., Priya, T. S., e Muneeswaran, K. (2019). Smart pso-based secured scheduling approaches for scientific workflows in cloud computing. Soft. Comp., 23(5):1745-1765.

Tawfeek, M. A. e AbdulHamed, A. A. (2018). Service flow management with multiobjective constraints in heterogeneous computing. In ICCES, pages 258-263. IEEE.

Teylo, L., de Paula Junior, U., Frota, Y., de Oliveira, D., e de A. Drummond, L. M. (2017). A hybrid evolutionary algorithm for task scheduling and data assignment of data-intensive scientific workflows on clouds. Future Gener. Comput. Syst., 76:1-17. 